\title{
Universiteit
}

Leiden

The Netherlands

\section{Social identity threat and performance motivation : the interplay between ingroup and outgroup domains}

Derks, B.

\section{Citation}

Derks, B. (2007, February 22). Social identity threat and performance motivation : the interplay between ingroup and outgroup domains. Kurt Lewin Institute Dissertation Series. Kurt Lewin Instituut, Amsterdam. Retrieved from https://hdl.handle.net/1887/10080

Version: $\quad$ Not Applicable (or Unknown)

License: $\quad$ Licence agreement concerning inclusion of doctoral thesis in the Institutional Repository of the University of Leiden

Downloaded from: https://hdl.handle.net/1887/10080

Note: To cite this publication please use the final published version (if applicable). 


\section{Chapter 2 Social creativity strikes back: Social identity protection and performance motivation ${ }^{1}$}

When members of a devalued group are outperformed by a higher status outgroup on important status-defining dimensions, social identity theory proposes that they can reduce social identity threat by valuing alternative dimensions on which intergroup comparison is more positive (a form of 'social creativity', Lemaine, 1974; Tajfel \& Turner, 1986). For instance, women who perceive that men outperform them in their mathematical ability can claim superiority in terms of verbal skills, a dimension on which women stereotypically outperform men. This focus on alternative dimensions is usually viewed as a cognitive rather than a behavioral strategy (Van Knippenberg \& Ellemers, 1990). It is expected to improve the perception that low status group members have of their group and is seen to increase well-being without addressing the actual status difference between groups (Blanz, Mummendey, Mielke, \& Klink, 1998; Crocker \& Major, 1989; Mummendey \& Schreiber, 1984; Spears \& Manstead, 1989; Wright, 2001b). However, we propose that social creativity (by valuing alternative dimensions) can "strike back". That is, attaching value to an alternative ingroup dimension not only benefits stigmatized group members' well-being, but also stimulates individual group members' motivated performance on dimensions on which the group has low status. In three experiments, we examined whether valuing an alternative dimension in addition to a status-defining dimension enhanced individual low status group members' status improvement behavior.

\section{Advantages and disadvantages of social creativity}

Van Knippenberg and Ellemers (1990) introduced the term 'social cooperation' to describe a situation in which groups each claim superiority on one dimension, while acknowledging the outgroup's superiority on another dimension. Although the term social cooperation refers to the potential to foster positive 
intergroup relations, the strategy of focusing on alternative dimensions to deflect social identity threat is expected to have negative effects for low status group members. Specifically, focusing on alternative dimensions on which the ingroup excels can lead group members to cognitively devalue and disidentify from statusdefining dimensions on which group performance is low, while these latter dimensions continue to define the social hierarchy (Crocker \& Major, 1989; Crocker, Major, \& Steele, 1998; Major \& Schmader, 1998; Osborne, 1995). Following theories of motivation such as expectancy-value theory, which define motivation as dependent on the value attached to a dimension, domain disidentification is expected to damage the motivation to succeed on that dimension (Atkinson \& Birch, 1978). For instance, it is unlikely that ethnic minorities will gain more favorable economic outcomes if their focus on alternative dimensions of ingroup superiority such as sports or music keeps them from investing in dimensions necessary for economic success such as academic achievement.

On the one hand then, focusing on alternative dimensions is a positive strategy that reduces threat to stigmatized group members' social identity and enhances their well-being (Cadinu \& Cerchioni, 2001; Ellemers \& Van Rijswijk, 1997). On the other hand, when the focus on alternative ingroup dimensions leads individuals to ignore and devalue status-defining dimensions, use of this strategy may dissuade stigmatized group members from attempts to improve their group's standing on the status-defining dimension, hence preserving existing intergroup status differences (Crocker \& Major, 1989; Osborne, 1995). From this point on, we focus on the perspective of the low status group, using the term ingroup dimensions to refer to alternative dimensions that are identified by devalued groups to enhance social identity. We use the term outgroup dimensions to refer to dimensions on which the high status group's superior standing is based.

\section{Can social creativity increase motivation on outgroup dimensions?}

The central hypothesis in this chapter is that when members of a devalued group attach value to ingroup dimensions (social creativity), this actually protects their motivation on outgroup dimensions. When group members experience a threat to their social identity, they show psychological and physiological stress responses (Matheson \& Cole, 2004). Because threat can stand in the way of motivated performance on outgroup dimensions, this threat needs to be resolved in order for members of devalued groups to remain focused on outgroup dimensions. Indications for this have been found in self-affirmation research that focuses on personal 
identity threat. For example, Kurman (2003) showed that self-affirmation after individual failure increases self-improvement behavior in the domain in which one failed. Moreover, Sherman and Cohen (2002) showed that an opportunity to selfaffirm reduces defensive responses to failure, hereby increasing the opportunity for individuals to improve performance. In this chapter we propose that affirming social identity similarly benefits motivation on outgroup dimensions. However, some strategies to cope with stress resulting from social identity threat (e.g., domain devaluation) are more likely to undermine motivation than others (i.e., valuing ingroup dimensions). In contrast to earlier accounts of social creativity, we thus propose that stigmatized group members who deflect social identity threat by valuing ingroup dimensions can successfully cope with the stress that social identity threat poses, enabling them to become motivated to perform on outgroup dimensions. Importantly, whereas previous research has mostly focused on effects of social identity protection on the well-being of members of devalued groups, we take a new approach by examining how valuing alternative dimensions affects performance motivation on status-relevant outgroup dimensions.

In sum, we predict that in situations in which the need for social identity protection is high and group members experience stress, attaching value to ingroup dimensions alleviates stress and protects motivation on outgroup dimensions. Importantly however, we predict that valuing ingroup dimensions will only be beneficial for motivation when the outgroup dimension remains valued as well. Thus, in contrast to earlier conceptions of social creativity predicting low motivation on status-relevant dimensions when people turn to alternative dimensions, we aim to show that social creativity can actually bolster motivation as long as it does not lead to devaluation of the outgroup dimension.

\section{The present studies}

In three studies, we investigate whether members of devalued groups who are in threatening intergroup situations, will display higher motivation on outgroup dimensions when they can protect social identity by valuing an ingroup dimension. In Experiments 1 and 2, we create intergroup settings and employ different manipulations to manipulate identity threat and the degree to which the ingroup dimension is already implicitly valued. Within these contexts, we examine whether motivation on an outgroup dimension is pronounced when devalued group members protect their social identity by personally valuing an ingroup dimension. By examining contexts that differ in the degree to which they elicit threat and 
implicitly value the ingroup dimension, we assess whether the positive relation between personally valuing ingroup dimensions on motivation is especially high when social identity threat is high and the ingroup dimension is not already valued. In Experiment 1, we examine the effects of personally valuing ingroup dimensions on motivation to perform on the outgroup dimension in contexts in which either members of the ingroup (low threat) or the outgroup (high threat) are present. In Experiment 2, we examine this relationship in contexts that differ in how salient the status difference between the groups is (low and high threat) and how available the ingroup dimension is within that context (low and high implicit value of the ingroup dimension). In Experiment 3, we induce social identity threat and offer social identity protection by manipulating the explicit value of outgroup and ingroup dimensions expressed by others. That is, we examine whether a context in which others value the outgroup dimension (social identity threat) as well as the ingroup dimension (social identity protection) protects social identity and personal well-being while at the same time motivating group members to increase performance on the outgroup dimension.

We obtain support for our predictions in experimentally created minimal groups (in Experiments 1 and 2) as well as in an existing low status group (women in Experiment 3). All three studies use experimentally created intergroup settings in which participants are members of a group that is assigned low status on a (fictitious) status-relevant dimension and high status on a (fictitious) alternative dimension. In this way we ensured that participants had no previous experience with the dimensions so that we were able to credibly manipulate performance feedback. Moreover, to ensure that participants were focused on their social identity instead of their personal identity, all three experiments were explicitly framed as looking at intergroup performance differences in which participants were addressed as members of the ingroup under investigation.

\section{Experiment 1}

Experiment 1 manipulated an intergroup context in which participants' ingroup was assigned low status. We examined whether coping with threat by personally valuing an ingroup dimension benefited motivation to perform on the outgroup dimension under conditions of social identity threat (low/high). We studied this relationship in two contexts: one context in which only ingroup members were present and one in which only outgroup members were present. As will be shown in Chapter 3 of this dissertation (Derks, Van Laar, \& Ellemers, 2006a), 
outgroup contexts are perceived as threatening by stigmatized group members because these contexts are expected to place emphasis on the outgroup dimension. Ingroup contexts are less threatening as they are expected to place emphasis on the ingroup dimension. We predict that in outgroup contexts, individuals who attach high value to an ingroup dimension will become more motivated on the outgroup dimension. In ingroup contexts, social identity threat will be low as the ingroup dimension is already perceived as important (Derks et al., 2006a). Therefore, in this context valuing the ingroup dimension is not expected to predict motivation on the outgroup dimension. In other words, we predict that the effect of valuing the ingroup dimension on motivation on the outgroup dimension is moderated by group context (Hypothesis 1).

We hypothesize that the degree to which group members value the outgroup dimension will be less predictive of motivation on the outgroup dimension in contexts that already stress the importance of this dimension (i.e., outgroup contexts). Thus, we expect that in outgroup settings personally valuing the outgroup dimension is less predictive of motivation on this dimension compared to ingroup settings in which more contextual emphasis is placed on the ingroup dimension (Hypothesis 2).

\section{Method}

\section{Design and participants}

Eighty-four Leiden University students were randomly assigned to one of two conditions (ingroup or outgroup context). Participants voluntarily participated and were paid the equivalent of five Euros. Nine students were removed: five because they failed to complete the test measuring group status, and four because they spontaneously reported in a thought-listing-task that they suspected that the status feedback was manipulated. The remaining 75 students (19 males) had a mean age of 21 years $(\mathrm{SD}=2.01)$.

Procedure

Participants were seated in separate cubicles and received instructions via a computer. Participants read that the purpose of the study was to examine differences in 'mental flexibility' (allegedly the skill to oversee complex problems and to come up with ingenious solutions for these problems) between detailed and global perceivers. Mental flexibility was introduced as a status-relevant dimension by telling participants that this skill was unrelated to general intelligence but highly predictive of career success. All participants were then assigned to the group of 
detailed perceivers supposedly on the basis of Gerard and Hoyt's (1974) dotestimation task. In this task, participants were asked to estimate the number of dots that appear on the screen and were classified as detailed perceivers (who underestimated the number of dots) or global perceivers (who overestimated the number of dots). We created an intergroup context by showing participants that among the twelve participants that were (supposedly) present, six were detailed perceivers (the ingroup) and six were global perceivers (the outgroup). Then, we administered a bogus mental flexibility test consisting of a word-formation task in which participants were to form new words out of a ten-letter word, and a multiplechoice task in which series of four figures were to be completed with a fifth figure.

Induction of low group status: To create a status hierarchy in which detailed perceivers received low status, following the mental flexibility test participants read that previous studies had established that global perceivers (the outgroup) generally outperformed detailed perceivers (the ingroup) on this test. The rationale provided for this was that global perceivers were more able to oversee a situation (the letters or figures in the mental flexibility test) and therefore performed better than detailed perceivers, who focused more on details. However, we provided participants with an ingroup dimension characteristic of detailed perceivers, namely 'stepwise thinking'. In concordance with detailed perceivers' focus on details, this dimension was introduced as the ability to think about the details and consequences of existing solutions to problems, hereby fine-tuning these solutions. It was explicitly stated that this dimension was less predictive of career success and different from mental flexibility. Participants read that in previous studies ingroup members (detailed perceivers) were found to perform better on this dimension than outgroup members (global perceivers). Participants did not gain experience with the ingroup dimension themselves and did not receive personal feedback on stepwise thinking. Participants were then shown how all 12 participants in the session had performed on the mental flexibility test. In this way it was conveyed that the scores of all group members present (including themselves) reflected the intergroup performance difference that was supposedly found in other studies. Personal performance feedback prevented participants from protecting their self-esteem by distancing themselves from low ingroup performance by estimating their personal performance as higher than the group's performance (Schmader, Major, Eccleston, \& McCoy, 2001).

Manipulation of group context: Participants read that an oral mental flexibility test would follow which they would take in the presence of five other 
participants. In the ingroup condition these other participants were all said to be detailed perceivers, and in the outgroup condition these participants would all be global perceivers. We checked the effectiveness of this manipulation in conveying that the others present at the oral test would be likely to perform well on the outgroup dimension with six items ( $\alpha=.82$, e.g., 'Among the people with whom I'm about to take the oral test, the mean mental flexibility is very low/ very high').

Following this manipulation, to further reinforce the significance of mental flexibility as a status-defining dimension, we set up a lottery for five prizes of 20 Dutch guilders, in which participation required a high score (60) on the outgroup dimension. Participants were subsequently informed that it was also possible to take an oral version of a test measuring the ingroup dimension of stepwise thinking. However, to communicate the lesser relevance of this dimension participants were told that if they chose to do this test, a higher score (80) had to be obtained in order to receive a lottery ticket. Also, we conveyed that it was less self-evident that participants might use this option, because participants had to write an e-mail to the research assistant if they wanted to do this test. This was done to reinforce the notion that the outgroup dimension was the primary determinant of material outcomes, whereas the ingroup dimension was the alternative dimension.

During the experiment, participants were asked quiz-questions checking their understanding of the critical manipulations (e.g., whether they understood the higher importance to career success of the outgroup dimension relative to the ingroup dimension, whether they correctly identified their group membership). After measuring the dependent variables, we asked participants to list the thoughts they had during the experiment to check whether they spontaneously indicated suspicion concerning the manipulations. Then, instead of performing the oral test, participants were debriefed, thanked and paid for their cooperation. After finishing data collection, the five prizes were awarded by lottery.

Measures

All dependent variables were measured on nine-point Likert-type scales. We checked whether participants felt more identified with their minimal ingroup than the minimal outgroup with two five-item scales measuring identification with the ingroup and the outgroup ( $\alpha_{\text {ingroup }}=.93$, $\alpha_{\text {outgroup }}=.87$, e.g. ' 'I feel connected to the group of detailed/global perceivers', and 'The group of detailed/global perceivers is important to me'). We checked whether group context indeed affected identity concerns in two ways. First, three items measured how threatening participants thought it would be to have to perform the oral mental flexibility test in the 
presence of other participants and how threatened and tense they felt as a result of the feedback they received on the mental flexibility test $(\alpha=.83)$. Second, we measured state self-esteem with a shortened (six item) version of the Rosenberg selfesteem scale (Rosenberg, 1965, $\alpha=.90$ ) that we adapted to measure state selfesteem. ${ }^{2}$ Personal value attached to the ingroup and the outgroup dimension was measured with three items for each dimension $\left(\alpha\right.$ ingroup dimension $=.78$, $\alpha_{\text {outgroup dimension }}=$ .82 , 'I think mental flexibility/stepwise thinking is an important ability', 'How good I am in mental flexibility/stepwise thinking is important to me', 'It does not matter whether my mental flexibility/stepwise thinking ability is high or low'[reverse coded]). Motivation to increase performance on the outgroup dimension was measured using three items ( $\alpha=.89$, 'I want to work hard to obtain higher mental flexibility', 'I am not going to put effort in trying to achieve higher mental flexibility'[reverse coded], 'I would like to practice to develop my mental flexibility').

\section{Results}

Table 2.1 lists the correlations between all dependent variables.

Table 2.1. - Means (SD) and Correlations Between Dependent Variables in Experiment 1

\begin{tabular}{lccccc}
\hline & $\begin{array}{c}\text { Mean } \\
(S D)\end{array}$ & 1 & 2 & 3 & 4 \\
\hline 1. Threat & 5.16 & & & & \\
& $(1.89)$ & & & & \\
2. Self-esteem & 6.77 & $-.53^{* *}$ & & & \\
& $(1.48)$ & & & & \\
3. Value outgroup dimension & 6.35 & .07 & $-.21^{\dagger}$ & & \\
& $(1.49)$ & & & & \\
4. Value ingroup dimension & 6.23 & -.02 & -.02 & $.48^{* *}$ & \\
& $(1.57)$ & & & & \\
5. Motivation outgroup dimension & 5.65 & -.08 & -.17 & $.57^{* *}$ & $.56^{* *}$ \\
& $(1.90)$ & & & & \\
\hline
\end{tabular}

Note: ${ }^{\dagger} p<.1,{ }^{*} p<.01,{ }^{* *} p<.001$ 


\section{Manipulation checks}

Manipulation checks of group context indicated that, compared to participants in the outgroup condition, participants in the ingroup condition expected the other participants with whom they would take the oral test to perform less well on mental flexibility $\left(M_{\text {ingroup }}=4.02, S D=.95 ; M_{\text {outgroup }}=6.93, S D=1.15\right)$, $F(1,73)=144.75, p<.001$, partial $\eta^{2}=.67$.

As intended, participants recognized detailed perceivers as their ingroup. A repeated-measures ANOVA on group identification (ingroup/outgroup) by group context indicated that participants indeed felt more identified with their ingroup ( $M$ $=4.35, S D=1.96)$ than with the outgroup $(M=2.83, S D=1.36), F(1,73)=41.10, p<$ .001 , partial $\eta^{2}=.36$. Identification was not affected by group context $(F=1.03)$.

Threat

As intended, participants in the outgroup condition felt more threatened about their past performance and the upcoming oral mental flexibility test $(M=$ 5.63, $S D=2.10)$ than participants in the ingroup condition $(M=4.75, S D=1.59)$, $F(1,73)=5.22, p=.04$, partial $\eta^{2}=.06$.

State self-esteem

Participants who anticipated an interaction with members of the outgroup reported lower state self-esteem $(M=6.38, S D=1.73)$ than participants who were anticipating an interaction with members of the ingroup $(M=7.10, S D=1.15)$, $F(1,73)=4.65, p=.03$, partial $\eta^{2}=.06$. This indicates that group context did indeed result in identity threat.

Personal value of ingroup and outgroup dimensions

Group context did not affect the degree to which participants personally valued the outgroup dimension $(M=6.35, S D=1.49, F<1)$. The value participants attached to the ingroup dimension, however, was higher in the ingroup context ( $M$ $=6.57, S D=1.60)$ than in the outgroup context $(M=5.84, S D=1.47), F(1,73)=4.71$, $p<.05$, partial $\eta^{2}=.05$.

Motivation on outgroup dimension

Motivation on the outgroup dimension was unaffected by group context $\left(M_{\text {ingroup }}=5.86, S D=1.93, M_{\text {outgroup }}=5.42, S D=1.86, F=1\right)$.

Personal value as a moderated predictor of motivation on the outgroup dimension

Using hierarchical multiple regression and analyzing simple slope (Aiken \& West, 1991), we tested the degree to which motivation on the outgroup dimension was related to personal value attached to the ingroup or the outgroup dimension in 
the ingroup condition compared to the outgroup condition (see Table 2.2). We examined whether group context moderated the degree to which personal value attached to the ingroup and the outgroup dimension predicted motivation. First, we standardized the continuous variables. Then we performed hierarchical multiple regression analysis in which the main effects of group context ( 1 = ingroup, $2=$ outgroup) and personal value attached to the ingroup and outgroup dimension were entered in step 1, and the two-way interactions between these variables and the hypothesized moderator group context were entered in step 2 . Step 1 revealed that, as predicted, both valuing the ingroup dimension $(B=.36, S E=.10, p<.001$, semipartial $\left.r^{2}=.09\right)$, and valuing the outgroup dimension $(B=.40, S E=.10, p<.001$, semi-partial $r^{2}=.12$ ) were associated with higher motivation on the outgroup dimension.

Table 2.2. - Hierarchical Regression of Motivation on the Outgroup Dimension in Experiment 1

\begin{tabular}{lllll}
\hline & $\begin{array}{l}\mathrm{R}^{2} \\
\text { Change }\end{array}$ & $B$ & $S E$ & $\begin{array}{l}\text { Semi- } \\
\text { partial } r^{2}\end{array}$ \\
\hline Step 1 & $.43^{* * *}$ & & & \\
$\quad$ Group Context & & -.06 & .19 & .00 \\
$\quad$ Value OD & & $.40^{* * *}$ & .10 & .12 \\
$\quad$ Value ID & & $.36^{* *}$ & .10 & .09 \\
Step 2 & $.05^{*}$ & & & \\
$\quad$ Value OD x Group Context & & $-.56^{*}$ & .21 & .05 \\
$\quad$ Value ID x Group Context & & .31 & .21 & .02 \\
\hline
\end{tabular}

Note: ${ }^{*} p<.05,{ }^{* *} p<.01,{ }^{* * *} p<.001$

The important question is whether the manipulation of group context moderates the relationship between value attached to the ingroup and outgroup dimension and motivation on the outgroup dimension. Confirming Hypothesis 2 , the interaction between group context and value attached to the outgroup dimension was significant ( $B=.56, S E=.21, p=.01$, semi-partial $r^{2}=.05$ ). Thus, whether valuing an outgroup dimension was associated with higher motivation on this outgroup dimension depended on whether in- or outgroup members were present in the context. Although the hypothesized interaction effect between group 
context and value attached to the ingroup dimension did not reach significance (Hypothesis 1, $B=.31, S E=.21, p=.14$, semi-partial $r^{2}=.02$ ), to test our prediction we performed planned simple slope analyses separately for the ingroup and the outgroup condition. As predicted, the results show that in the outgroup context motivation on the outgroup dimension was positively related to the degree to which participants' personally valued the ingroup dimension $(B=.54, S E=.16, p=.001$, semi-partial $\left.r^{2}=.09\right)$, but unrelated to the degree to which they valued the outgroup dimension $(B=.03, S E=.17, p=.85)$. By contrast, in a context in which only members of the ingroup were present, motivation to perform on the outgroup dimension was predicted by personal value attached to the outgroup dimension ( $B=$ .59, $S E=.12, p<.001$, semi-partial $r^{2}=.17$ ), but less by personal value attached to the ingroup dimension $\left(B=.22, S E=.14, p=.11\right.$, semi-partial $\left.r^{2}=.02\right)$. Thus, supporting Hypothesis 1 , in a context that induced social identity threat (i.e., the outgroup context) valuing the ingroup dimension was related to higher motivation on the outgroup dimension. However, value attached to the ingroup dimension was not related to motivation in a context in which social identity was protected (i.e., the ingroup context). In addition, supporting Hypothesis 2, in the ingroup context motivation on the outgroup dimension was predicted by the degree to which participants refrained from devaluing the outgroup dimension. However, in the outgroup context value attached to the outgroup dimension did not predict motivation on this dimension.

\section{Discussion}

This study examined whether valuing a dimension on which the ingroup is successful is positively related to individual group members' motivation on an outgroup dimension. The results confirmed our central hypothesis that valuing an ingroup dimension protects motivation on the outgroup dimension when social identity is threatened. In an outgroup context in which social identity threat results in lower well-being (as was indicated by higher reported threat and lower selfesteem), personally valuing an ingroup dimension is positively related to performance motivation on the outgroup dimension. By contrast, in an ingroup context (in which social identity concerns are less salient) motivation on the outgroup dimension is predicted by how highly individuals value the outgroup dimension. Moreover, personal value attached to the outgroup dimension was less related to motivation on the outgroup dimension in an outgroup context, likely 
because the importance of performing well on the outgroup dimension was already sufficiently salient in that context (see also Chapter 3 of this dissertation).

Experiment 1 manipulated social identity threat through the presence of ingroup and outgroup members. Experiment 2 focused on the ingroup context and manipulated social identity threat by varying the salience of the intergroup status difference. Additionally, we directly manipulated opportunities to improve social identity by varying how readily available the ingroup dimension was as an alternative performance dimension. Again, we examined under which conditions personally valuing an ingroup dimension positively predicted motivation to perform on the outgroup dimension.

\section{Experiment 2}

Firstly, we manipulated social identity threat by varying the contextual salience of the status difference in terms of the relative performance of the two groups. We predicted that in contexts in which low group status is highly salient, valuing an ingroup dimension is related to higher motivation on the outgroup dimension. However, in contexts in which the status difference is less salient, social identity threat should be less pronounced, and the motivation to perform on the outgroup dimension will depend solely on the personal value attached to the outgroup dimension (Hypothesis 1).

Secondly, we manipulated the ease with which social identity was protected by manipulating the contextual availability of the ingroup dimension. In a context in which the ingroup dimension is readily available, for example when female students are in a setting in which their verbal skills are considered useful, social identity needs are easily fulfilled. In these contexts motivation on the outgroup dimension will therefore be predicted mostly by personal value attached to the outgroup dimension. By contrast, in contexts in which the ingroup dimension is less available, for instance for female students in a setting that only evaluates their math performance, stigmatized group members need to personally value the ingroup dimension to protect social identity before they can focus on the outgroup dimension (Hypothesis 2).

In addition, we theorized that the moderating effects of availability of the ingroup dimension would be especially apparent under high social identity threat (i.e., high salience of the status difference). Thus, Hypothesis 3 predicted two threeway interactions between the experimental factors and personal value attached to the ingroup and the outgroup dimension. 


\section{Method}

Design and participants

Experiment 2 followed a 2 (status difference salience low/high) X 2 (availability of the ingroup dimension low/high) design. We recruited 131 students from the University of California, Los Angeles and randomly assigned them to one of the four experimental conditions. Participants voluntarily participated in return for course credit. Three participants were excluded from our analysis because their answers on one of the two manipulation checks of status difference salience indicated that they had not understood this manipulation. The remaining sample of 128 participants (52 males) had a mean age of 19 years $(S D=3.02)$.

Procedure

The procedure was similar to Experiment 1 with respect to assigning participants to the group of detailed (vs. global) perceivers, inducing an intergroup status difference based on a performance difference in mental flexibility, and providing participants with an ingroup dimension on which their group's performance was superior (stepwise thinking). This time only five other ingroup participants (detailed perceivers) were (supposedly) present.

Manipulation of salience of status difference: Salience of the status difference was increased by showing participants the higher mean performance of previous outgroup participants. Thus, all participants were informed about the generally found performance difference between detailed and global perceivers in mental flexibility, the mean performance (50) of the ingroup in the present study, and about their personal performance (49). However, only participants in the high salience condition were also informed about the average performance for an outgroup of global perceivers (88) from their university. We checked this manipulation by measuring the perceived performance difference between the groups (e.g., 'What do you think is the average score of detailed perceivers/global perceivers on the mental flexibility test?') and by measuring the time needed to estimate the outgroup's performance.

Next, participants read that they would be orally tested on the outgroup dimension (mental flexibility) in the presence of the five other ingroup participants. We informed them of the possibility to choose the alternative stepwise thinking test instead of the mental flexibility test. Identical to Experiment 1, participants could 
earn lottery tickets for five prizes of 20 US Dollars with high performance on mental flexibility.

Manipulation of availability of the ingroup dimension: The ingroup dimension was made more easily available by giving participants an option to take an oral test measuring the ingroup dimension instead of the outgroup dimension. All that participants in the high availability condition needed to do was to indicate which of the two tests they wanted to take. In the low availability condition the ingroup dimension was made less available by indicating that when participants wanted to take the alternative test, they were required to write an e-mail to the research assistant. As a manipulation check, we examined whether participants were more likely to choose the alternative test when it was more easily available. After measuring the dependent variables, participants were debriefed and paid for their cooperation. Upon completion of the study, the five prizes were awarded by lottery. Measures

All items were identical to those in Experiment 1. Again, identification with the ingroup $(\alpha=.90)$ and outgroup $(\alpha=.92)$ was measured. Also, state selfesteem was measured both before the experiment (presented as an unrelated study, $\alpha=.86)$ and after the experiment $(\alpha=.85)$ to examine whether group context and availability of the ingroup dimension affected identity concerns. Moreover, we measured personal value attached to ingroup $(\alpha=.78)$ and outgroup dimensions $(\alpha=$ .79), and participants' motivation to improve performance on the outgroup dimension (two items, $r=.71$, 'I want to work hard to obtain higher mental flexibility', 'I would like to practice to develop my mental flexibility').

\section{Results}

Table 2.3 lists the correlations between the dependent variables. ${ }^{3}$

\section{Manipulation checks}

Manipulation checks of status difference salience showed that our manipulation had the intended effect. Repeated-measures ANOVA showed that, although participants in both salience conditions perceived a performance difference between the groups on the outgroup dimension, $F(1,126)=292.39, p<.001$, partial $\eta^{2}=.70$, participants in the high salience condition perceived a larger status difference than participants in the low salience condition, $F(1,126)=6.47, p=.01$, partial $\eta^{2}=.05$. Moreover, participants in the high salience condition needed less time to estimate the performance of outgroup members $(M=6.71, S D=3.80)$ than participants in the low salience condition $(M=8.60, S D=4.68), F(1,126)=6.31, p$ 
$=.01$, partial $\eta^{2}=.05$, indicating that the high performance of the outgroup was more cognitively available to them.

As intended, the manipulation of contextual availability of the ingroup dimension affected the likelihood that participants opted for the alternative test. More participants in the high availability condition (23 out of 64 participants) opted for the alternative test than in the low availability condition (9 out of 64 participants), $\chi^{2}(1, N=128)=8.17, p=.01$. This choice was not affected by the salience of status difference manipulation, $\chi^{2}(1, N=128)=.01, p=1 .{ }^{4}$

Finally, a repeated-measures ANOVA on group identification (ingroup/outgroup) by group context showed that, in each condition (all $F \mathrm{~s}<1$ ), participants felt more identified with their ingroup $(M=4.77, S D=1.65)$ than with the outgroup $(M=3.62, S D=1.44), F(1,124)=50.82, p<.001$, partial $\eta^{2}=.29$.

Table 2.3. - Means (SD) and Correlations Between Dependent Variables in Experiment 2

\begin{tabular}{lcccccc}
\hline & Mean & 1 & 2 & 3 & 4 & 5 \\
& $(S D)$ & & & & & \\
\hline 1. Pre self-esteem & 7.27 & & & & & \\
& $(1.08)$ & & & & & \\
2. Post self-esteem & 6.65 & $.60^{*}$ & & & & \\
& $(1.23)$ & & & & & \\
3. Value outgroup dimension & 5.96 & -.08 & -.06 & & & \\
& $(1.59)$ & & & & & \\
4. Value ingroup dimension & 6.10 & -.01 & -.03 & $.83^{*}$ & & \\
& $(1.53)$ & & & & & \\
5. Motivation outgroup dimension & 5.72 & -.10 & -.10 & $.64^{*}$ & $.66^{*}$ & \\
& $(1.81)$ & & & & & \\
6. Test choice (1 = OD, 2 = ID) & 1.25 & .01 & -.01 & .05 & .00 & .08 \\
& $(.43)$ & & & & & \\
\hline
\end{tabular}

Note: $^{*} p<.001$

State self-esteem

Although neither manipulation had as strong an effect on personal selfesteem as group context had in Experiment 1, interactively they marginally affected 
self-esteem. An ANCOVA on post-manipulation state self-esteem controlling for pre-experimental state self-esteem, showed a marginally significant interaction between the two experimental factors, $F(1,123)=2.85, p=.09$, partial $\eta^{2}=.02$. When the salience of the status difference was low, self-esteem was not affected by the degree to which the ingroup dimension was available (Mless avalable $=6.67, S E=.17$; $\left.M_{\text {highly available }}=6.58, S E=.18, F<1\right)$. However, when the status difference was highly salient, participants reported higher self-esteem when the ingroup dimension was readily available $(M=6.93, S E=.17)$, than when this dimension was less available $(M=6.43, S D=.18), F(1,125)=4.19, p=.04$, partial $\eta^{2}=.03$. No other simple effects were statistically significant. Thus, only when the status difference between the groups was highly salient, did the availability of the ingroup dimension predict selfesteem.

Personal value of the outgroup and ingroup dimension

Across conditions, participants attached equal value to the outgroup dimension $(M=5.96, S D=1.59$, all $F \mathrm{~s}<1)$ and the ingroup dimension $(M=6.10, S D$ $=1.53$, all $F \mathrm{~s}<2$ ).

Motivation on the outgroup dimension

Motivation to increase performance on the outgroup dimension was lower when the status difference was not as salient $(M=5.35, S D=1.73)$ than when it was highly salient $(M=6.10, S D=1.82), F(1,124)=5.71, p<.02$, partial $\eta^{2}=.04$. Thus, participants were less motivated to increase their performance on the outgroup dimension, when they had not seen the details of the intergroup performance difference on the test. This finding seems to contradict social identity theory predicting decreased motivation on outgroup dimensions resulting from social identity threat. However, we interpret this result not so much as increased motivation when salience is high, but as decreased motivation when salience is low. Since participants in the low salience condition only saw test-scores of themselves and ingroup members, but not of outgroup members, this could have lowered the necessity of improving performance.

Personal value as a moderated predictor of motivation on the outgroup dimension

We again used hierarchical multiple regression to examine the degree to which personally valuing ingroup and outgroup dimensions was related to higher motivation on the outgroup dimension in the four conditions. Specifically, we examined whether the salience of the status difference and the availability of the ingroup dimension moderated these relationships. First, we standardized the continuous scales. Then, in step 1, we regressed motivation on test choice, status 
difference, availability of the ingroup dimension and personal value attached to each of the dimensions. In step 2, we added the two-way interactions between the experimental factors and personal value attached to each dimension. Finally, in step 3 , two three-way interactions between the two experimental manipulations and personal value attached to each dimension were entered (see Table 2.4).

Table 2.4. - Hierarchical Regression of Motivation on the Outgroup Dimension in Experiment 2

\begin{tabular}{lllll}
\hline & $\mathrm{R}^{2}$ Change & $B$ & $S E$ & $\begin{array}{l}\text { Semi } \\
\text { partial } r^{2}\end{array}$ \\
\hline Step 1 & $.49^{* * *}$ & & & \\
$\quad$ Test choice & & .12 & .15 & .00 \\
Salience & $.29^{*}$ & .13 & .02 \\
Availability ID & -.06 & .13 & .00 \\
Value OD & $.31^{* *}$ & .11 & .03 \\
Value ID & & $.38^{* *}$ & .12 & .04 \\
Step 2 & & & \\
$\quad$ Value OD x Salience & & -.02 & .25 & .00 \\
$\quad$ Value ID x Salience & & .33 & .25 & .01 \\
$\quad$ Value OD x Availability & & -.38 & .24 & .01 \\
$\quad$ Value ID x Availability & & $.51^{*}$ & .24 & .02 \\
$\quad$ Salience x Availability & & -.18 & .26 & .00 \\
Step 3 & .01 & & & \\
$\quad$ Value OD x Salience x & & -.18 & .50 & .00 \\
$\quad$ Availability & & & & \\
$\quad$ Value ID x Salience x & & -.27 & .51 & .00 \\
$\quad$ Availability & & & & \\
\hline Note: ${ }^{\dagger} p<.1^{*} p<.05,{ }^{* *} p<.01,{ }^{* * *} p<.001$ & &
\end{tabular}

In step 1 , both personal value attached to the ingroup dimension $(B=.38$, $S E=.12, p<.01$, semi-partial $\left.r^{2}=.04\right)$ and personal value attached to the outgroup dimension $\left(B=.31, S E=.11, p<.01\right.$, semi-partial $\left.r^{2}=.03\right)$ significantly predicted motivation on the outgroup dimension. Moreover, higher salience of the status difference was associated with higher motivation on the outgroup dimension ( $B=$ 
.29, $S E=.13, p<.03$, semi-partial $r^{2}=.02$ ). A statistically significant interaction in step 2 between availability of the ingroup dimension and personal value attached to the ingroup dimension partly confirmed Hypothesis 1, showing that whether the ingroup dimension was available moderated the degree to which valuing the ingroup dimension was associated with higher motivation on the outgroup dimension $\left(B=.41, S E=.24, p<.05\right.$, semi-partial $\left.r^{2}=.02\right)$. Salience of the status difference did not reliably moderate the effects of personally valuing the ingroup dimension (nor the outgroup dimension) on motivation on the outgroup dimension. Moreover, step 3 did not reveal significant three-way interactions. Thus, contradicting Hypothesis 3, availability of the ingroup dimension moderated the effects of personally valuing the ingroup and outgroup dimensions on motivation independent of the salience of the status difference.

We subsequently examined the moderating effect of availability of the ingroup dimension. Although the interaction between personally valuing the outgroup dimension and availability of the ingroup dimension did not reach statistical significance (Hypothesis 2, $B=-.38, S E=.24, p=.11$, semi-partial $r^{2}=.01$ ), we inspected the simple slopes of personally valuing the ingroup dimension and the outgroup dimension in the two conditions that differed in availability of the ingroup dimension. When the ingroup dimension was available as a performance dimension, motivation on the outgroup dimension was higher when participants attached more value to the outgroup dimension $\left(B=.52, S E=.16, p<.01\right.$, semi-partial $\left.r^{2}=.04\right)$. As predicted, in this condition motivation on the outgroup dimension was unrelated to personal value attached to the ingroup dimension $(B=.08, S E=.18, p=.49)$. By contrast, as expected, when the ingroup dimension was less available, motivation on the outgroup dimension was higher when participants were more inclined to value the ingroup dimension $\left(B=.59, S E=.16, p<.001\right.$, semi-partial $\left.r^{2}=.06\right)$. In this condition motivation on the outgroup dimension was unrelated to personal value attached to the outgroup dimension $(B=.12, S E=.17, p=.47)$.

\section{Discussion}

Experiment 2 provides further support for our central prediction that, in contexts in which stigmatized group members experience social identity threat, valuing ingroup dimensions is related to higher motivation to perform well on an outgroup dimension. Although the salience of the status difference did not affect this relation, the availability of the ingroup dimension did moderate whether valuing ingroup dimensions predicted higher motivation on the outgroup 
dimension. In a context that emphasizes the value of the outgroup dimension by implying that performance is restricted to this dimension, low status group members' motivation on the outgroup dimension benefits from social identity protection by valuing the ingroup dimension. However, in a context in which the ingroup dimension is already implicitly valued (as communicated by the possibility to choose this dimension instead of the outgroup dimension), valuing the outgroup dimension is related to higher motivation on the outgroup dimension.

In contrast to Experiment 1, in Experiment 2 we were unable to affirm that the experimental manipulations indeed affected identity threat as indicated by global self-esteem. Participants' global self-esteem was only affected by availability of the ingroup dimension when the status difference between the groups was highly salient. However, since both manipulations were designed to affect social identity, it is conceivable that personal self-esteem was not the best indicator of this type of identity threat. Possibly, more direct measures of social identity threat, such as collective self-esteem (Luhtanen \& Crocker, 1992), would have revealed that making the ingroup dimension available indeed affirms social identity. Moreover, the manipulations in Experiment 2 seem to be more subtle compared to the manipulations of ingroup/outgroup context in Experiment 1, making it less likely to find effects on an overall self-esteem measure.

Whether the ingroup dimension was contextually available moderated the relations between personally valuing the ingroup and outgroup dimension and motivation on the outgroup dimension, but salience of the status difference did not. Thus, for members of a group that has already received low status, the degree to which the ingroup dimension is available seems to be a more important factor in predicting whether social identity protection is positively related to motivation than the exact degree to which social identity is threatened. Similarly, in Experiment 1, the manipulation of ingroup/outgroup context not only affected identity threat but is also expected to affect the degree to which participants perceive the ingroup dimension to be valued (Derks et al., 2006a, see Chapter 3 of this dissertation). These results suggest that personal value attached to the ingroup and outgroup dimension is only essential for social identity protection and motivation on the outgroup dimension when the contextual value for these dimensions is somewhat ambiguous. Contextual value for an ingroup dimension seems to provide social identity protection. In Experiment 3 we therefore examined the effects of contextual value for the ingroup and outgroup dimension to examine whether a context that emphasizes the importance of both dimensions indeed protects social identity and 
enhances self-improvement motives on the outgroup dimension at the same time. Moreover, by manipulating social identity protection directly by varying the contextual value that is accorded to the ingroup dimension, we can test whether contextual value attached to the ingroup dimension indeed causes higher motivation on the outgroup dimension.

Experiments 1 and 2 relied on self-report measures of performance motivation. It remains to be seen whether the reported motivation on the outgroup dimension translates into observable differences in persistence and performance on the outgroup dimension. In Experiment 3 we therefore went beyond self-report measures of motivation and examined actual persistence and performance on the outgroup dimension.

\section{Experiment 3}

Experiment 3 investigated the impact of the contextual value of ingroup and outgroup dimensions on the motivation and performance of stigmatized group members on the outgroup dimension. We tested whether a context that emphasizes the importance of both ingroup and outgroup dimensions can reduce social identity concerns at the same time that it motivates stigmatized group members to increase performance on the outgroup dimension. Contextual value of the two dimensions was orthogonally manipulated. We measured affective threat to see whether the experience of threat (implying the need to protect social identity) was higher when the outgroup dimension was contextually important, and to check whether feelings of threat were indeed diminished by raising the contextual value of the ingroup dimension (Hypothesis 1). Furthermore, we included a behavioral measure of performance motivation by assessing the time that low status group members invested in a task relevant to the outgroup dimension as well as their actual performance on this task. We predicted persistence and performance to be highest when both the ingroup and the outgroup dimension were contextually valued (Hypothesis 2). Finally, to extend the insights obtained in Experiment 1 and 2, Experiment 3 examined responses of members of an existing low status group, namely female students in an experiment modeled on a job-application context. In the Netherlands, women often occupy low status positions in the labor market, there are few women in executive positions and females receive lower pay than do male employees (Portegijs, Boelens, \& Olsthoorn, 2004). 


\section{Method}

Design and participants

The experiment followed a 2 (contextual value outgroup dimension low/high) X 2 (contextual value outgroup dimension low/high) design. Participants were 91 female Leiden University students $\left(M_{a g e}=20, S D=3.52\right)$. Originally, 110 participants participated. Based on a thought-listing task, 19 participants were excluded, as they spontaneously indicated that they suspected the feedback about their performance on the two dimensions was preprogrammed. ${ }^{5}$ Participants were randomly assigned to one of four experimental conditions and received 5.5 Euros for their voluntary participation.

Procedure

Participants were seated in separate computer cubicles and received all information via the computer. Participants read that they were participating in a study on gender performance differences on two tests that were used in assessment centers to evaluate job candidates. Two tests were administered supposedly measuring distinct cognitive abilities: 'creative integration' and 'inferential flexibility'. Creative integration functioned as the outgroup dimension and was described as the ability to see logical connections between concepts that initially seem incompatible. Inferential flexibility functioned as the ingroup dimension and was described as the ability to quickly oversee a situation while simultaneously paying attention to all aspects. The test that measured creative integration (the outgroup dimension) was adapted from McFarlin and Blascovich's Remote Associates Test (RAT, 1984) in which participants are shown three words (for instance 'elephant', 'lapse' and 'vivid') and are to suggest a fourth word that is related to all three words (in this case 'memory') as quickly as possible. In the 'Word Fragment Test' measuring inferential flexibility (the ingroup dimension), the participants were shown only vowels and were to add consonants to form an existing word, choosing out of three given alternatives. Their task was to answer 40 items correctly in as short a time as possible.

Manipulation of contextual value of the two dimensions. To vary the contextual value of each dimension, participants were informed that not all cognitive abilities were equally valued by employers and that therefore not all tests were administered equally often in real assessment centers. In the low contextual value condition, participants were told that the (ingroup or outgroup) test was not used very often because employers did not think this ability was of high value. In the high contextual value condition, participants were told that the (ingroup or 
outgroup) test was used very often because employers regarded this ability of high value. We checked this manipulation with three items for each dimension ( $\alpha_{\text {ingroup }}$ dimension $=.86$, $\alpha$ outgroup dimension. $=.91$ e.g., 'How important do you think it is for a successful career to have high creative integration ability?').

Status manipulation: Participants received pre-programmed feedback and learned that their performance on the creative integration test (the outgroup dimension) fell in the category 'below average' and that their performance on the inferential flexibility test (the ingroup dimension) fell in the category 'above average'. Participants were informed that this was in line with earlier research that had established that in general men tended to outperform women on creative integration, but that women tend to outperform men on inferential flexibility. This induction was checked with four items measuring the perceived performance of men and women on the outgroup dimension ('Within the domain of creative integration women/men perform 1 [very badly] - 9[very well]'). Finally, we checked whether participants felt identified with the female gender group by measuring agreement with six items measured on nine-point scales $(\alpha=.85$, e.g., 'It is important to me to be a women', 'I feel commitment towards other women'). After the dependent measures were administered, participants were debriefed, thanked and paid for their participation.

Measures

Threat experienced as a result of the performance outcomes was measured by asking how threatened, well (reverse coded), restless, insecure and frustrated participants felt as a result of the status feedback they received (nine-point scales, $\alpha$ $=.88$ ). Persistence and performance on the outgroup dimension were assessed during a second administration of the RAT in which participants could stop whenever they wanted. We assessed the time participants worked on this test and the number of items they completed correctly (corrected for performance on the first RAT).

\section{Results}

Table 2.5 lists the correlations between the dependent variables. Manipulation checks

As intended, when the outgroup dimension had high contextual value participants perceived this dimension to be more important for a successful career $(M=7.23, S D=1.31)$ than when the outgroup dimension had lower contextual value $(M=3.49, S D=1.31), F(1,87)=183.15, p<.001$, partial $\eta^{2}=.68$. Likewise, when the ingroup dimension had high contextual value participants perceived this dimension 
to be more important for career success $(M=7.29, S D=1.51)$, than when the ingroup dimension had low contextual value $(M=3.23, S D=1.19), F(1,87)=201.26$, $p<.001$, partial $\eta^{2}=.70$. There were no other significant main effects or interactions. Participants indeed felt identified with the female gender group $(M=6.78$, $S D=1.09$ ), and this identification was not affected by the experimental manipulations (all $F \mathrm{~s}<1$ ).

Finally, to exclude the possibility that any effects of our manipulations on the dependent variables were caused by a change in participants' perception of the intergroup status difference on the outgroup dimension, we checked whether the manipulations of contextual value affected the perceived performance difference between men and women on the outgroup dimension. We calculated a performance difference score by subtracting the estimated performance of women on the outgroup dimension from the estimated performance of men. An ANOVA indicated that participants in all four conditions perceived the performance of men on the outgroup dimension as higher than that of women $(M=3.98, S D=1.59$, all $F \mathrm{~s}<.1)$. Thus, contextual value for both dimensions did not increase motivation on the outgroup dimension because it decreased the perceived intergroup performance difference on the outgroup dimension.

Table 2.5. - Means (SD) and Correlations Between Dependent Variables in Experiment 3

\begin{tabular}{lccc}
\hline & $\begin{array}{c}\text { Mean } \\
(S D)\end{array}$ & 1 & 2 \\
\hline 1. Threat & 3.65 & & \\
& $(1.46)$ & & \\
2. Persistence on outgroup dimension & 15.18 & -.03 & \\
& $(5.49)$ & & \\
3. Performance on outgroup dimension & 11.11 & -.07 & $.73^{*}$ \\
& $(5.70)$ & & \\
\hline
\end{tabular}

Note: ${ }^{*} p<.001$

\section{Threat}

As predicted in Hypothesis 1, higher affective threat was experienced when the outgroup dimension had high contextual value $(M=4.03, S D=1.49)$ than when 
it had low contextual value $(M=3.26, S D=1.33), F(1,87)=6.94, p=.01$, partial $\eta^{2}=$ .07 (see Figure 2.1). This effect was qualified by an interaction, $F(1,87)=5.31, p=$ .02 , partial $\eta^{2}=.06$. As predicted, when the outgroup dimension had high contextual value, knowing that the ingroup dimension was also contextually valued reduced threat $(M=3.50, S D=1.34$ ) compared to when the ingroup dimension was not valued $(M=4.53, S D=1.48), F(1,88)=6.21, p=.015$, partial $\eta^{2}=.07$. Contextual value of the ingroup dimension did not affect threat when the outgroup dimension had low contextual value ( $\left.M_{\text {low }}=3.10, S D=1.29 ; M_{\text {high }}=3.41, S D=1.38 ; F<1\right)$. Moreover, when the ingroup dimension was not valued, high contextual value of the outgroup dimension led to higher threat than low contextual value of this dimension, $F(1,88)$ $=12.39, p<.01$, partial $\eta^{2}=.12$. Contextual value of the outgroup dimension, however, did not affect threat when the ingroup dimension was contextually valued $(F<1)$.

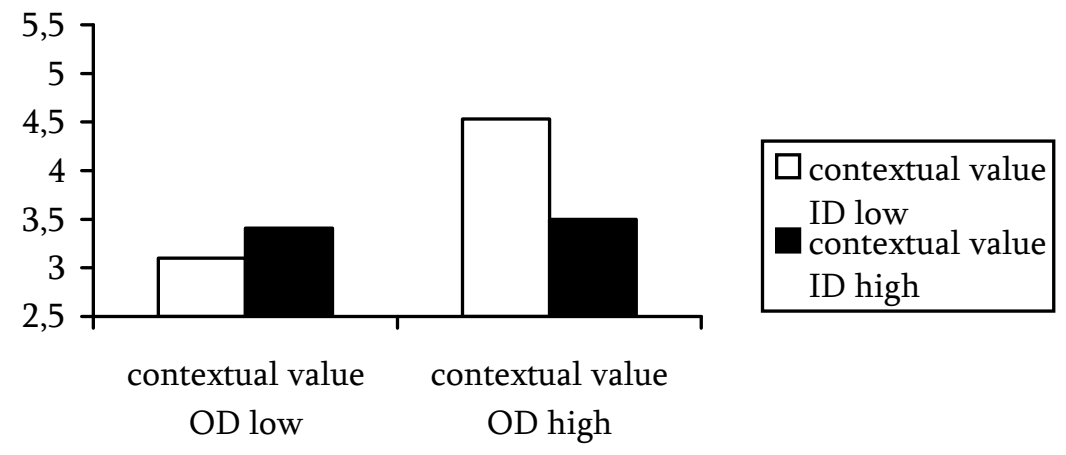

Figure 2.1. Perceived threat under low and high contextual value of the ingroup (ID) and the outgroup $(O D)$ dimension in Experiment 3.

\section{Motivation and performance on the outgroup dimension}

Because the time participants voluntarily spent working on the second administration of the outgroup task was not normally distributed, we transformed this variable by taking its square root. As predicted in Hypothesis 2, highest motivation and performance was found when both dimensions were contextually valued. On persistence, we found a significant interaction only, $F(1,87)=7.56, p<$ .01 , partial $\eta^{2}=.08$ (see Figure 2.2). When the outgroup dimension was contextually 
valued, valuing the ingroup dimension resulted in higher persistence on the outgroup dimension $(M=17.85, S D=6.04)$ than not valuing this dimension $(M=$ $13.60, S D=5.08), F(1,88)=7.51, p<.01$, partial $\eta^{2}=.08$. However, when the outgroup dimension had low contextual value, the contextual value of the ingroup dimension did not reliably affect persistence on the outgroup dimension $\left(M_{\text {low }}=\right.$ 15.62, $\left.S D=3.67 ; M_{h i g h}=13.75, S D=5.94\right), F(1,88)=1.43, p=.23$. Also, when the ingroup dimension was valued, persistence was higher when the outgroup dimension was equally valued, than when it was valued less, $F(1,88)=6.87, p=.01$, partial $\eta^{2}=.07$.

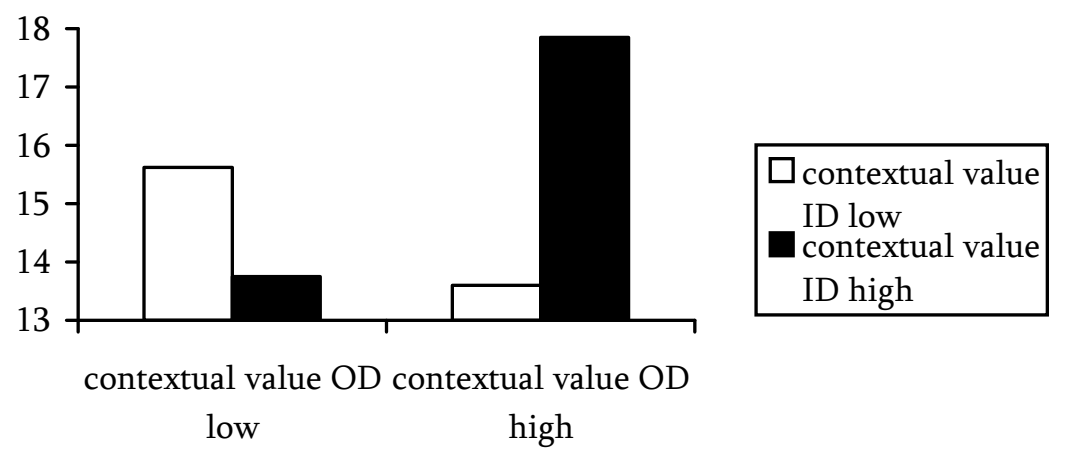

Figure 2.2 - Persistence (transformed time) on the outgroup dimension under low and high contextual value of the ingroup (ID) and the outgroup dimension (OD) in Experiment 3.

Higher persistence was accompanied by higher performance on the outgroup dimension. Again, we found a significant interaction only, $F(1,86)=5.59, p$ $<.03$, partial $\eta^{2}=.06$ (see Figure 2.3). When the outgroup dimension was highly valued, performance was higher when the ingroup dimension was valued as well (adjusted $\left.M_{\text {low }}=9.72, S E=1.08 ; M_{\text {high }}=13.25, S E=1.10\right), F(1,88)=5.30, p<.03$, partial $\eta^{2}=.06$. However, when the outgroup dimension was not valued, whether the ingroup dimension was valued did not affect performance (adjusted $M_{\text {low }}=11.65$, $\left.S E=1.16 ; M_{h i g h}=9.93, S E=1.10\right), F(1,88)=1.23, p=.27$. In addition, when the ingroup dimension was valued, higher performance was found when the outgroup dimension was valued as well, $F(1,88)=4.30, p<.04$, partial $\eta^{2}=.08$. When the 
ingroup dimension was not valued, performance was not affected by the contextual value of the outgroup dimension, $F(1,88)=1.62, p=.21$.

Because motivated performance was unrelated to affective threat (see Table 2.5), the higher motivation resulting from contextual value for both dimensions could not be attributed to reduced threat.

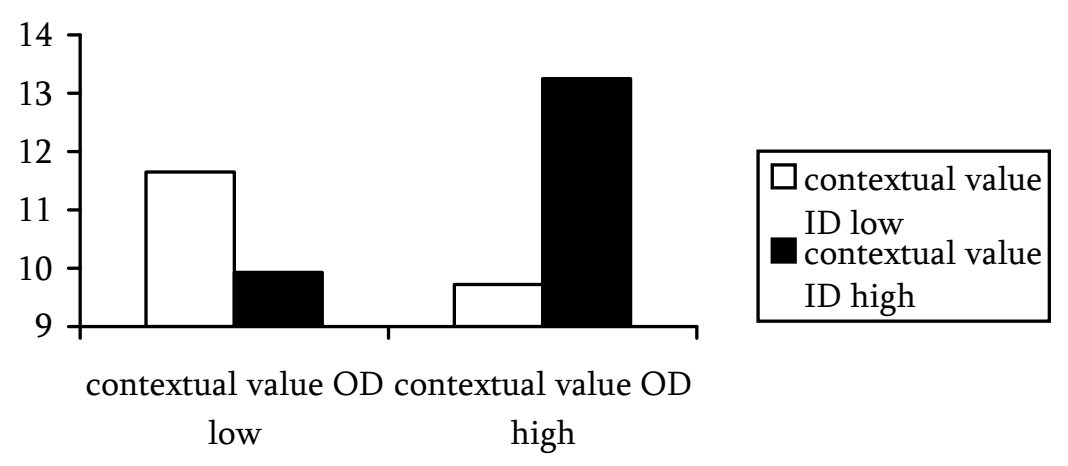

Figure 2.3 - Performance on the outgroup dimension under low and high contextual value of the ingroup (ID) and the outgroup dimension (OD) in Experiment 3.

\section{Discussion}

The results of Experiment 3 complement the results of Experiments 1 and 2. Experiments 1 and 2 show that when social identity is threatened, protecting social identity by personally valuing the ingroup dimension is positively related to selfreported motivation on the outgroup dimension. Experiment 3 shows that when both ingroup and outgroup dimensions are contextually valued, low status group members display highest motivation on the outgroup dimension. Importantly, Experiment 3 extended the results of the first two studies in that it revealed the predicted effects on actual task behavior. That is, members of an existing low status group (women in an employee assessment context) showed higher task persistence and performance on the outgroup dimension when both ingroup and outgroup dimensions were contextually valued, compared to when only the outgroup dimension was valued. 
In addition, Experiment 3 provided further insight into the psychological process hypothesized to be associated with this effect. The results show that the personal experience of threat is indeed influenced by situational cues with regard to the value of ingroup and outgroup dimensions. When low status group members perceive the outgroup dimension on which they previously failed to be contextually relevant, they experience more threat than when the outgroup dimension was deemed unimportant in that context. Furthermore, we found that increasing the contextual value of an ingroup dimension on which ingroup performance is higher than that of the high status group alleviates this threat and results in selfimprovement behavior, as is evident from higher motivated performance on the outgroup dimension. However, the non-significant correlation between affective threat and motivated performance indicates that, although both are affected by contextual value of the ingroup and outgroup dimensions, the reduction in threat did not directly account for increased motivated performance on the outgroup dimension.

\section{General Discussion}

The research reported here examined the beneficial effects of social creativity by valuing ingroup dimensions on low status group members' motivation and performance on (status-defining) outgroup dimensions. The results from three experiments converge to show that when low status group members are in situations in which outgroup dimensions are perceived as important, valuing ingroup dimensions (either personally or contextually) reduces their feelings of threat, and promotes their motivation, persistence and performance on outgroup dimensions. We showed that when multiple dimensions are available, engagement in social creativity by valuing ingroup dimensions not only enhances social identity and alleviates threat, as was previously assumed (Lemaine, 1974; Tajfel \& Turner, 1986), but also elicits actual status-improving behavior. Thus, the use of this form of social creativity as a cognitive strategy to alleviate threat is not limited to affective outcomes such as well-being, as was previously assumed, but actually prepares group members for self-improvement behavior.

Importantly, this effect only emerges when the status-defining dimension also remains valued. When social creativity results in devaluation of the outgroup dimension, these beneficial effects do not occur. In this sense, our experiments show that both dimensions need to be valued for these beneficial motivational effects to occur. Our results show that motivation and performance on an outgroup dimension 
depend on whether the value that is accorded to this outgroup dimension stimulates self-improvement motives. At the same time, motivation and performance on an outgroup dimension is a function of whether low status group members are able to reduce the stress resulting from their low status by perceiving the ingroup dimension as valued. This stress is reduced in contexts in which the ingroup dimension is already valued or group members can reduce it themselves by focusing on the ingroup dimension. When neither of these preconditions is met (e.g., because the situation does not emphasize one of these dimensions and the individual's personal value attached to this dimension is low) motivation on the outgroup dimension is reduced. In Experiments 1 and 2, the induction of social identity threat indirectly manipulated whether contextual emphasis was placed on either the ingroup or the outgroup dimension. The results from these experiments indicated that motivation to improve performance on the outgroup dimension was highest among participants who balanced the high contextual value of one dimension with a high personal value attached to the other dimension. In Experiment 3, we orthogonally manipulated the contextual value of both dimensions, showing that the highest motivation and performance on the outgroup dimension is observed in a context that attaches value to both dimensions. Attesting to the robustness of these findings, we obtained these converging results in support of our argument with different group types (experimental groups and natural groups), different tasks and methodologies and in different socio-cultural contexts (the Netherlands in Experiment 1 and 3, and the United States in Experiment 2).

The results of these three experiments have important implications for the status improvement of stigmatized group members such as women and ethnic minorities in real world settings (work and school environments). Our results indicate that in order to motivate stigmatized group members to perform on dimensions that are important for social status - such as employment and education value must be given to dimensions that are important to the devalued group. When the context only emphasizes how important it is to perform well on dimensions on which high status groups excel (e.g., academic achievement), this increases social identity threat among devalued groups, leading members of these groups to become less motivated to improve on these dimensions and encouraging them to withdraw and focus on ingroup dimensions (e.g., sports, family relations). In contrast, by endorsing the value of dimensions on which devalued groups excel, social identity is protected so that motivation on the outgroup dimension can be maintained or enhanced. This suggests, for example, that by communicating value for ethnic or 
religious backgrounds, such as Islam and ethnic identities, ethnic minority students may come to feel that their group membership is valued, and that they can strive for high academic performance irrespective of the negative stereotypes of their group within this domain. Similarly, emphasizing the value of domains of importance to women could lead women in traditionally male-dominated fields to feel less threatened about the status of their gender group and would motivate them to optimize their achievement at work and pursue a career in these fields. The possibility to protect social identity is especially important in contexts, such as the Netherlands, in which a high percentage of women work in lower level part-time jobs and stop working when they start a family (Portegijs, et al., 2004).

The results also emphasize low status group members' need for a distinctive subgroup identity that is valued by other subgroups in society, and have implications for the integration of ethnic minorities into multiethnic societies (Dovidio, Gaertner, \& Validzic, 1998; Hornsey \& Hogg, 2000; Mummendey \& Wenzel, 1999). Berry's acculturation model (Berry, 1997, 2001) argues that multicultural societies that explicitly value diversity allow ethnic minorities to participate in society while simultaneously maintaining their ethnic identity. Importantly, such integration benefits ethnic minorities' long-term health and well-being. Societies that communicate low regard for minorities, and that ask them to assimilate into the host society and to abandon their cultural background, induce minorities to segregate, resulting in suboptimal outcomes for both society in general (e.g., intergroup conflict) and minority group members in particular (e.g. health outcomes, wellbeing). Consistent with this, Huo and Molina (2006) showed that in a pluralistic society such as the United States, perceiving acknowledgement of and respect towards one's subgroup leads ethnic minorities to identify with the common identity (i.e., Americans) and to have more trust in the justice system. Our research shows that similar processes affect motivated performance. This suggests that a society that communicates respect towards subgroups by valuing the dimensions that they value not only enhances well-being and the attitude towards this society, but also increases their motivation to perform well on the dimensions that define status in this society. Of course, low status group members within societies that devalue ingroup dimensions can still protect their motivation by personallyvaluing ingroup dimensions. However, personally valuing ingroup dimensions is just one strategy out of a range of social creativity strategies group members can choose to protect social identity, among which some are very detrimental to motivation. In the long run, the increase in motivation resulting from contextually affirming low 
status group members' social identity can diminish the status difference between low and high status groups.

\section{Limitations and suggestions for further research}

One important question that deserves further attention concerns the exact process that accounts for the higher motivation and performance on outgroup dimensions that we observed in situations that emphasize both ingroup and outgroup dimensions. We proposed that social identity concerns result in a state of affective threat that stands in the way of motivated performance. Therefore, this threat needs to be addressed in order for motivation on outgroup dimensions to be maintained. The results of Experiment 3 indeed show that self-reported threat was lower when ingroup and outgroup dimensions were contextually important, indicating that social identity concerns were addressed. However, threat was uncorrelated with motivation and performance on the outgroup dimension. Thus, although a context in which both ingroup and outgroup dimensions are seen as important is both more motivating and less threatening, we were unable to show that the reduction of threat in itself accounted for the higher motivation on the outgroup dimension. One possibility is that additional aspects of self-enhancement (that is, other than self-reported levels of threat) are important for self-improvement to emerge. In a current research project not discussed in this dissertation we are assessing more unobtrusive aspects of threat that can be assessed with the physiological measures of Blascovich and colleagues (Blascovich, Mendes, Hunter, \& Salomon, 1999; Blascovich \& Tomaka, 1996). In addition, future research might examine other possible mediators for the self-improvement effects we observed. One likely candidate is the perception of the performance situations as a challenge rather than a threat (Lazarus \& Folkman, 1984). We expect that contextual value for high performance of the ingroup on alternative dimensions may have increased participants' cognitive appraisal of the performance situation as a challenge. Although challenge and threat are often conceptualized as two extremes of one psychological concept, we expect that because challenge appraisals are more related to approach motivation than threat appraisals (which are more likely to induce a vigilant state of avoidance), challenge appraisals could be more predictive of motivated performance on outgroup dimensions. In Chapter 4 of this dissertation, we investigate whether increased challenge appraisals as a result of social identity enhancement mediate performance motivation on outgroup dimensions, with lowered threat emerging as a by-product of this motivational process. 
In addition to examining possible mediating processes that operate on the level of the individual self, future studies can more directly examine the role of social identity in motivated performance on outgroup dimensions. The experiments reported in this chapter employed alternative performance dimensions on which the ingroup outperformed the high status outgroup. Although these alternative dimensions were presented as unrelated to performance on the status-defining outgroup dimension, it is possible that these dimensions increased motivation because they were seen as relevant to perceived self-efficacy and performance on the outgroup dimension, increasing perceived self-efficacy on the outgroup dimension. Future studies can examine whether manipulations that more generally increase the perception that the ingroup is valued within the performance context (e.g., respect for the ingroup's norms and values) also increase devalued group members' motivated performance on outgroup dimensions. This would affirm our theoretical argument that it is in fact social identity threat and social identity enhancement that account for differences in motivated performance on the outgroup dimension among individual members of low status groups.

A third issue that deserves further examination in future research concerns the precise goals underlying the status-improving behavior that low status group members displayed. Low status group members can increase effort on an outgroup dimension for two reasons: to achieve higher personal status and show that they are as competent as any member of the high status outgroup (individual mobility, see Ellemers, 2001; Ellemers \& Van Laar, in press), or to collectively achieve higher group status by showing a superior group performance on the outgroup dimension (social change, see Wright, 2001b). One beneficial effect of enhancing social identity by valuing dimensions on which the ingroup excels is that it makes the group level salient. While both affirmation of the personal and social self may enhance motivation on the outgroup dimension, change to existing status hierarchies and improvement of group status is more likely when individual group members feel committed to their group and collectively strive for higher group status (Wright, 2001b). Valuing dimensions that are characteristic of the group thus allows devalued group members to increase their personal performance on status-defining dimensions at the same time as it maintains the salience of the group level necessary for social change for the group as a whole. We examine this issue in Chapter 4 of this dissertation. 


\section{Conclusion}

The results from these three experiments consistently show that ingroup dimensions are important sources of social identity enhancement that lead low status group members to improve their motivation and performance on outgroup dimensions. The insights emerging from this work redefine social creativity from a purely cognitive strategy that allows members of low status groups to protect social identity to a vehicle to maintain motivation on outgroup dimensions. These results show that social creativity can have important implications for status-improving behavior and thus "strike back" to reduce social inequality. 


\section{Footnotes}

${ }^{1}$ This chapter is based on Derks, Van Laar, \& Ellemers (in press).

${ }^{2}$ We used the following items: 'At this moment I take a positive attitude towards myself', 'At this moment I feel I have a number of good qualities', 'At this moment I am satisfied with myself', 'At this moment I certainly feel useless', 'At this moment I feel I do not have too much to be proud of', 'At this moment I am inclined to feel that I am a failure'.

${ }^{3}$ Although the correlation between value attached to both dimensions is high $(r=.83)$, collinearity diagnostics indicate that collinearity is not harming the interpretation of the regression analysis (Condition Index: outgroup dimension = 4.35 , ingroup dimension $=8.33$ ). Belsley, Kuh and Welsch $(1980)$ suggest that condition indices below 15 are not problematic. Most crucially, the relations between valuing the ingroup and outgroup dimension and motivation on the outgroup dimension are moderated by the availability of the ingroup dimension, indicating that each variable has a unique contribution in predicting motivation on the outgroup dimension.

${ }^{4}$ We controlled for the effects of test choice on reported motivation on the outgroup dimension in subsequent analyses, but whether participants chose to do the ingroup or outgroup test did not affect participants' general motivation to improve performance on the outgroup dimension (see Table 2.4). This suggests that participants chose to do the oral test measuring the ingroup dimension to avoid failing in the presence of others or because they were curious to learn about this test, rather than because they were unmotivated to increase performance on the outgroup dimension.

${ }^{5}$ This number is unusually high because prior to this experiment other researchers at Leiden University, using the same subject pool, had performed experiments using false feedback. As a result, some participants suspected that they received preprogrammed scores on the two tests. Because we found it essential for our experiment that participants believed their scores, we removed participants from the sample who spontaneously wrote down in the thought-listing-task administered as a suspicion check that they suspected that the feedback was manipulated. The 19 removed participants were equally distributed across the four conditions (Chi square $=.12, p=.55$ ) excluding the possibility that our conditions differed in credibility. Including all 110 participants resulted in similar and reliable, albeit smaller effects. Both the crucial interactions on reported threat, $F(1,106)=5.71, p<.03$, partial $\eta^{2}=$ 
.05 , and on persistence, $F(1,106)=4.51, p<.04$, partial $\eta^{2}=.04$, and performance, $F(1,105)=3.33, p=.07$, partial $\eta^{2}=.03$, remained (marginally) significant. 